\title{
Study on Remote Sensing Inversion of Atmospheric Water Vapor Content Based on FY3B/MERSI Data
}

\author{
Jinwen $\mathrm{Wu}^{1,2}$, Longyu Sun ${ }^{3}$, Yushu Zhang ${ }^{1,2}$, Ruipeng Ji ${ }^{1,2}$, WenyingYu ${ }^{1,2}$, Rui Feng ${ }^{1,2^{*}}$ \\ ${ }^{1}$ Shenyang Institute of Atmospheric Environment, China Meteorological Administration, Heping District, Shenyang, Liaoning, China \\ ${ }^{2}$ Key Laboratory of Agricultural Meteorological Disasters in Liaoning Province, Heping District, Shenyang, Liaoning, China \\ ${ }^{3}$ Shenyang Meteorological Bureau, Hunnan District, Shenyang, Liaoning, China
}

\begin{abstract}
Atmospheric water vapor plays a vital role in global climate change. It not only affects local weather and climate but also influences the global water cycle and energy balance. Therefore, an accurate understanding of the atmospheric water vapor content and its changes are of great importance for forecasting climate, understanding global climate change, and studying the greenhouse effect. In this paper, the inversion of atmospheric water vapor content was conducted by FY3B/MERSI data, and verification was made by comparing with the sounding data, ground water vapor pressure data, and AQUA/MODIS water vapor content data. The results show that FY3B/MERSI atmospheric water vapor content has a significant positive correlation with the sounding water vapor content, ground water vapor pressure data, and AQUA/MODIS water vapor content data. Among them, the correlation with the sounding water vapor content is the most significant. An inversion correction model of MERSI atmospheric water vapor content can be established to improve the inversion accuracy.
\end{abstract}

\section{Preface}

Water vapor is an important part of the atmosphere and is the source of precipitation ${ }^{[1]}$. The unique role of water vapor determines that water vapor can have an important impact on climate and weather. In many cases of severe convective weather, water vapor is one of the main drivers and can be considered as the carrier of convective weather ${ }^{[2]}$. It can affect not only the local weather and climate but also the global water cycle and energy balance through atmospheric circulation and phase change processes. An accurate understanding of the atmospheric water vapor content and its changes is of great significance for climate forecasting, understanding global climate change, and research on the greenhouse effect ${ }^{[3]}$. So far, there are five main methods for monitoring atmospheric water vapor content, which are radio-sounding technology, meteorological satellite observation, water vapor radiometer observation, radar detection technology, and ground hygrometer. Each method has its advantages, disadvantages, and limitations. The inversion of atmospheric water vapor content using satellite remote sensing data makes up for the disadvantage of spatial discontinuity. Kaufman and Gao et al. proposed the MODIS channel ratio algorithm in 1992, and their study showed that the use of the three-channel method based on the MODIS near-infrared (NIR) band could reach an accuracy of $13 \%{ }^{[4]}$ for the inversion of atmospheric water vapor content. In 2001, $\mathrm{Hu}$ et al. usedMODTRAN3.7, an atmospheric radiative transfer model, to simulate the constant coefficient of correlation between atmospheric transmittance and atmospheric water vapor for the $19(0.940 \mu \mathrm{m})$ channel and compared the inversion results with the sounding water vapor data ${ }^{[5]}$. In 2003, Gao et al. provided a comprehensive description of the method of inverting atmospheric water vapor content in the NIR bands and validated the accuracy of MODIS level 2 and 3 water vapor products ${ }^{[6]}$. In 2005, Mao et al. used NIR bands to invert the atmospheric water vapor content in the Jianghan area ${ }^{[7]}$. In 2010, Wu et al. used the two-channel ratio method and the three-channel ratio method to invert the atmospheric water vapor content in northeastern China and concluded that the two-channel method was more suitable for northeastern China ${ }^{[8]}$. In2013, Bennouna et al. used both NIR and thermal infrared bands to invert the atmospheric water vapor content in northeastern China ${ }^{[9]}$. In 2017, Wang performed a linear model correction for the water vapor values obtained using satellite inversion by means ofthe values obtained through GPS inversion, and the accuracy was improved by $49.1 \%{ }^{[10]}$. In 2019 , Liu et al. used GNSS data to correct the inversion model of atmospheric water vapor. Also, it was verified that the model could improve the accuracy of water vapor inversion ${ }^{[11]}$. Therefore, in this paper, the atmospheric water vapor content inversion is performed based on FY3B/MERSI data and the validation is made by comparing with the sounding data, ground water vapor pressure data, and AQUA/MODIS water vapor content data. 


\section{Data source}

FY3B/MERSI and EOS/AQUA sunny-weather satellite data in Liaoning were selected with the transit elevation angle greater than $30^{\circ}$ to avoid the influence of cloud radiation and geometric distortion. AQUA/MODIS data were obtained from the free data download provided by the official website of NASA. FY3B/MERSI data were obtained from the official website of China Satellite Data Service. In the end, the satellite transit data in 11 days, that is, June 19, September 5, and September 23 in 2011, August 13, May 5, and May 22 in 2012, June 13, October 3 , and October 9, October 16, October 30 in 2013, was used for inversion and comparison verification of the atmospheric water vapor content.

The sounding data of Shenyang sounding station at 08:00 on the corresponding date (N41 ${ }^{\circ} 41^{\prime}$, E123 ${ }^{\circ} 31^{\prime}$ ) was used. Also, a GTS1-2 digital sounding instrument was used for the measurement of each meteorological element. The water vapor pressure observation data of the ground meteorological station on the corresponding date was used for the comparison verification of the atmospheric water vapor content.

\section{Calculation method}

\subsection{Satellite Inversion of Atmospheric Water Vapor Content}

The theoretical basis of the satellite inversion of water vapor content is the NIR radiative transfer equation. The study shows that the atmospheric water vapor transmission rate is almost 1 in the $0.84-0.88 \mathrm{um}$, $0.99-1.07 \mathrm{um}$, and $1.22-2.16 \mathrm{um}$ band intervals and $0.88-0.99$ um is the atmospheric water vapor absorption band interval. Through a large number of experiments, Bore, Daniel Schoapfer and Klans J. Ittn et al. found that the ratio method for the atmospheric water vapor was feasible. Given this, the NIR radiative transfer equation was used to derive the two-channel ratio method and three-channel ratio method to invert the atmospheric water vapor content. The idea of both methods is to use the ratio of the reflectance of atmospheric water vapor absorption channel and atmospheric window channel to calculate the atmospheric water vapor transmission rate, and then invert the atmospheric water vapor content.

\subsubsection{Two-Channel Ratio Method}

Where $\omega_{\text {is }}$ the atmospheric water vapor content, and $\rho_{0.940}, \rho_{0.865}$ are the reflectance ratio of the $0.940 \mathrm{um}$ and $0.865 \mathrm{um}$ channel. FY3B/MERSI uses the two-channel ratio method to invert the atmospheric water vapor content.

\subsubsection{Three-Channel Ratio Method}

Where $\rho_{1.240}$ is the reflectance ratio of the 1.240 umchannel. AQUA/MODIS uses the three-channel ratio method to invert the atmospheric water vapor content.

\subsection{Calculation of Atmospheric Water Vapor Content from Sounding Data}

Where $e_{\text {is the saturation water vapor pressure, }} t_{d}$ is the dew point temperature, $\mathrm{g}_{\text {is }}$ the acceleration of gravity, $p_{0}$ is the ground pressure, $p_{1}$ is the pressure at the top of the sounding layer, and $R_{P}$ is the total water vapor content in the column (unit: $\mathrm{cm}$ ). Since most of the water vapor in the atmosphere is concentrated in the lower half of the troposphere, the water vapor content in the near-ground layer accounts for a large proportion of the water vapor content in the whole atmosphere. Considering this, the value of the water vapor content in the whole atmosphere calculated by means of the sounding station data is accurate. In the actual sounding data, the atmospheric water vapor content is not directly given, so we need to determine the value of water vapor pressure on each isobaric surface through the dew point according to the relationship between saturation water vapor pressure and temperature. Based on the ideal gas equation of state and thermodynamic functions, the relationship between saturation water vapor pressure and temperature can be derived. In practical applications, the empirical formula (Goff-Gratch equation) is often used. The atmospheric water vapor content can be calculated through the formula (3) on the basis of the pressure of each layer and the dew point temperature.

\section{Analysis of inversion results of atmospheric water vapor content}

\subsection{Relationship Between FY3B/MERSI and Sounding Atmospheric Water Vapor Content}

The water vapor content of the whole atmosphere is mainly concentrated at the bottom of the troposphere, and the sounding data terminate at about $30 \mathrm{~km}$ above the tropospheric height. Hence, the atmospheric water vapor content measured by satellite remote sensing and sounding is consistent on the vertical scale. The relationship between FY3B/MERSI and sounding atmospheric water vapor content shows (Figure 1) that there is a significant correlation between the two, with a correlation coefficient of 0.8552 and a p-value less than 0.001 . The atmospheric water vapor content of FY3B/MERSI inversion is generally $1.1 \mathrm{~cm}$ lower than that of the sounding on average with a minimum deviation of $0.4 \mathrm{~cm}$. A correction model for MERSI atmospheric water vapor content inversion can be established to improve the accuracy of the inversion. 


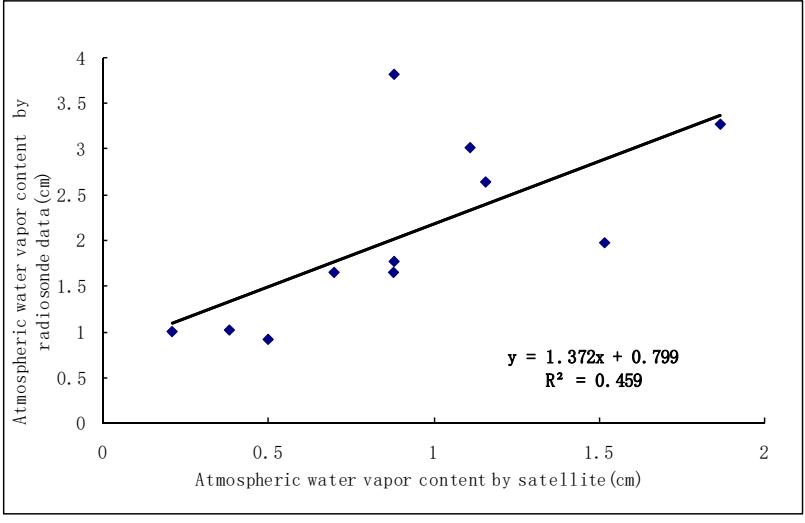

Figure 1. Relationship Between FY3B/MERSI and Sounding Atmospheric Water Vapor Content

\subsection{Relationship Between Atmospheric Water Vapor Content and Ground Water Vapor Pressure of FY3B/MERSI}

Since most of the atmospheric water vapor is concentrated in the lower half of the troposphere, the water vapor content of the whole atmosphere depends largely on the magnitude of the ground water vapor content, which should have a significant correlation with ground moisture parameters (such as water vapor pressure). The calculation results show that the atmospheric water vapor content has a highly significant correlation with the ground water vapor pressure of FY3B/MERSI inversion, with a correlation coefficient of $0.7367(\mathrm{n}=616)$ and a $\mathrm{p}$-value less than 0.001 (Figure 2). The spatial distribution maps were plotted by averaging the water vapor content of the 11-day sample data, and it was found that the trends of the spatial distribution maps of satellite atmospheric water vapor content and ground water vapor pressure also showed great consistency. There were two low-value areas of water vapor distribution in Jianping and Fuxin counties, and one high-value area of water vapor distribution in Donggang, Dandong (Figure 3 and Figure 4).

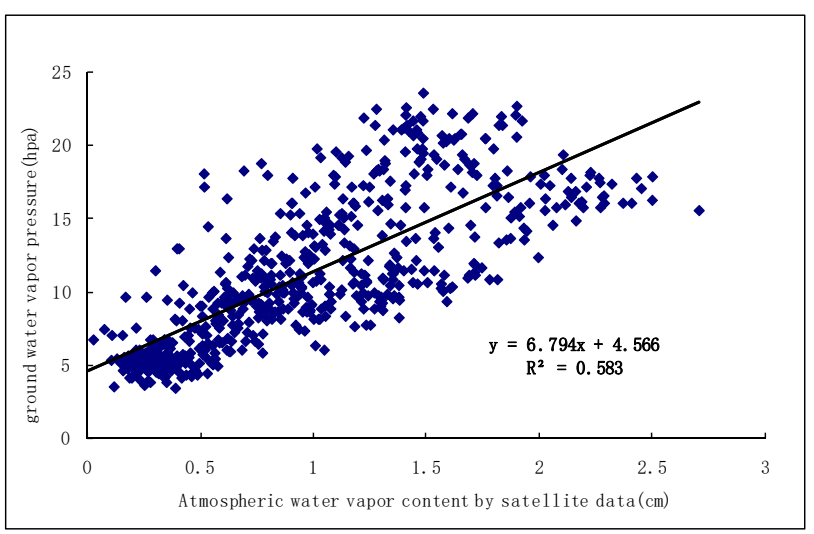

Figure 2 Relationship Between FY3B/MERSI Atmospheric Water Vapor Content and Ground Water Vapor Pressure

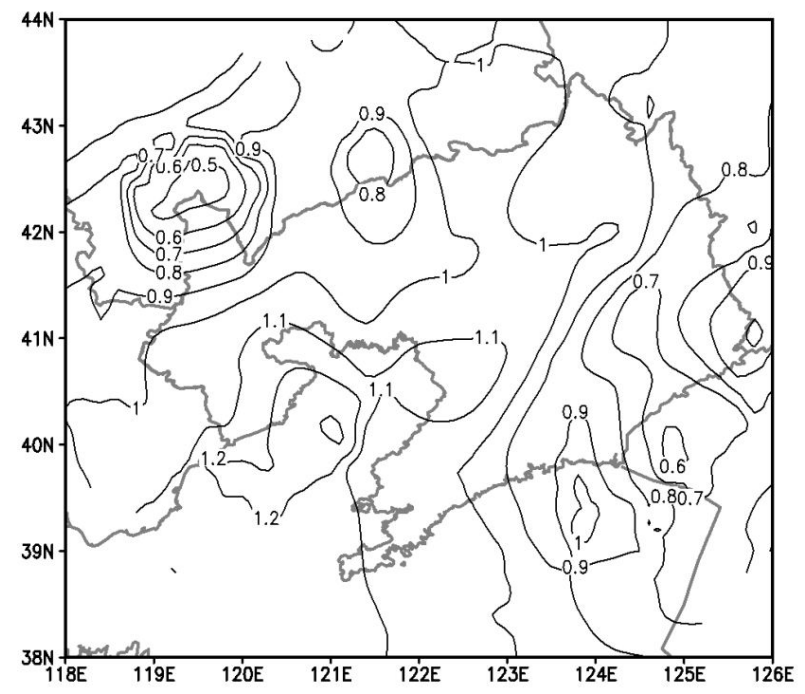

Figure 3 Spatial Distribution Map of FY3B/MERSI Atmospheric Water Vapor Content

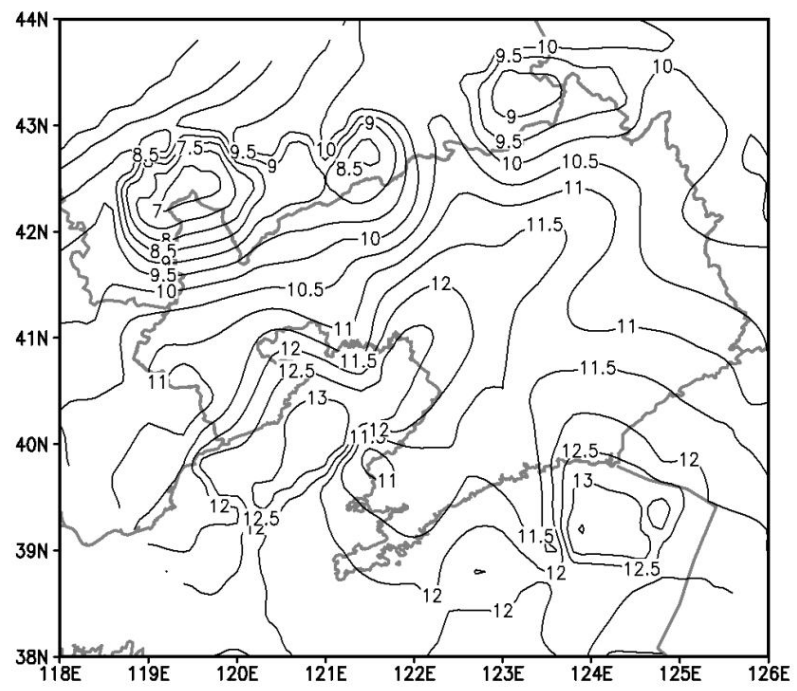

Figure 4 Spatial Distribution Mapof Ground Water Vapor Pressure

\subsection{Relationship Between FY3B/MERSI and AQUA/MODIS Atmospheric Water Vapor Content}

Based on the sample error analysis and satellite transit elevation angle, take the condition on May 22, 2013, which is close to the average error condition and has a high satellite elevation angle, as an example. The atmospheric water vapor content data of FY3B/MERSI and AQUA/MODIS in Liaoning were extracted. The results show that there is a highly significant correlation between them with a correlation coefficient of 0.8187 $(\mathrm{n}=9999)$. 


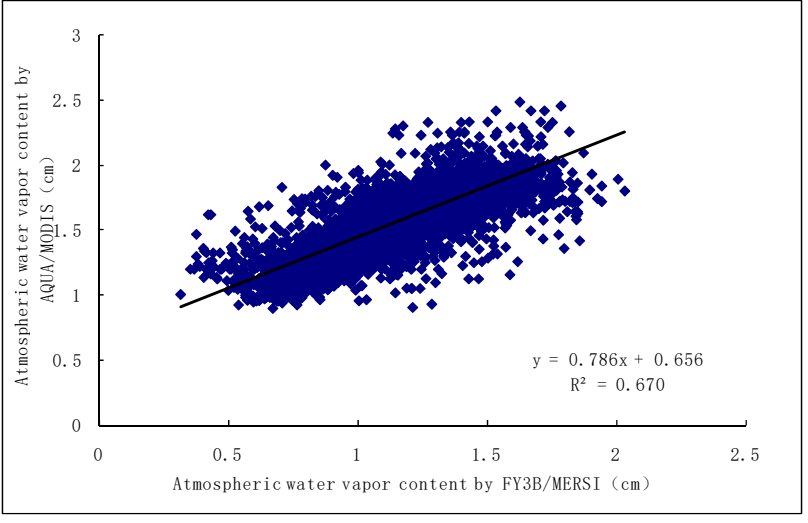

Figure 5 Relationship Between FY3B/MERSI and AQUA/MODIS Atmospheric Water Vapor Content

\section{CONCLUSION}

In this paper, the inversion of atmospheric water vapor content based on FY3B/MERSI data was performed andthe comparison with the sounding data, ground water vapor pressure data, and AQUA/MODIS water vapor content data was made. The following two conclusions were obtained.

(1) FY3B/MERSI atmospheric water vapor content has a significant positive correlation with sounding water vapor content, ground water vapor pressure data, and AQUA/MODIS water vapor content data.

(2) The correlation between FY3B/MERSI atmospheric water vapor content and sounding water vapor content is the most significant, and a correction model for MERSI atmospheric water vapor content inversion can be established to improve the inversion accuracy.

Since the distribution of atmospheric water vapor content has obvious seasonal characteristics, the relationship between FY3B/MERSI atmospheric water vapor content and sounding water vapor content, ground water vapor pressure data, and AQUA/MODIS water vapor content was not studied by season in this paper. Besides, the impact of latitude and longitude, land cover type, and topographic features on atmospheric water vapor content inversion was not considered.

\section{Project Funds}

Natural Science Fund Guide to Programs project of Liaoning Province (2019-ZD-0857); Key Project of Liaoning Provincial Meteorological Bureau (201603); Natural Fund Instruction Project of Liaoning Province (2019-ZD-0857); Key Project of Liaoning Meteorological Bureau (201603); Department of Science \& Technology of Liaoning Province (2018108004), and State Administration of Science. Technology and Industry for National Defense, PRC (70-Y40G09-9001-18/20).

\section{References}

1. Kepiao XU. A study on the characteristics of water vapor content and water vapor transport of the Tibetan Plateau[D]. University of Science and Technology of China,2020.

2. Emardson T, Webb F H. Estimating the motion of atmospheric water vapor using the Global Positioning System [J]. GPS Solutions,2002,58-64.

3. Haolin WEI. Research on Inversion of Precipitable Water Vapor Based on MODIS Data-Taking Gansu Province as an Example [D]. Lanzhou Jiaotong University,2020.

4. Kaufman Y J, Bo-Cai Gao. Remote Sensing of Water Vapor in the Near IR from EOS/MODIS[J]. IEEE Transactions on Geoscience and Remote Sensing, 1992, 30(5): 871-884.

5. $\mathrm{Hu}$ Xiuqing, Zhang Yuxiang, Huang Yifen, et al. Inversion of atmospheric column total water vapor using a solar radiometer with the $940 \mathrm{~nm}$ channel[J]. Meteorological Science and Technology, 2001, 29(3): 12-17.

6. Bo-Cai Gao, Kaufman Y J. Water vapor retrievals using Moderate Resolution Imaging Spectroradiometer (MODIS) near-infrared channels[J]. Journal of Geophysical Research, 2003, 108(13): 5-8.

7. Kebiao MAO, Zhihao QIN, Jianming WANG, etal. Low Tran Retrieval of an Atmospheric Water Content and Tran Smittance Computation of MODIS Bands 31 and 32[J]. Remote sensing for land and resources, 2005, 17(1): 26-29.

8. Junjie WU, Kaishan SONG, etal. MODIS-based Retrieval of Atmospheric Water Vapor content in Northeast China $[\mathrm{J}]$. Chinese Journal of Agrometeorology, 2010, 31(3): 447-452.

9. Bennouna Y S, Tomes B, Cachorro V E, et al. The evaluation of the integrated water vapor annual cycle over the Iberian Peninsula from EOS-MODIS against different ground-based techniques[J]. Quarterly Journal of the Royal Meteorological Society, 2013, 139(676): 1935-1956.

10. Xidi Wang. Research on Precipitable Water Vapor Based on GPS and MODIS Data[D]. Southeast University, 2017.

11. Bei LIU, Yong WANG, Zesheng LOU, etal. The MODIS PWV Correction Based on CMONOC in Chinese mainland[J]. Acta Geodaetica et Cartographoca Sinica, 2019, 48(10): 1207-1215. 